\title{
Guía de recomendaciones para el manejo de pacientes pediátricos con enfermedad severa por SARS-CoV-2*
}

\author{
Recommendations guide for the management of pediatric patients with severe \\ SARS-CoV-2
}

\author{
Christian Scheu Goncalves ${ }^{\mathrm{a}}$, Adriana Diettes González ${ }^{\mathrm{b}}$, Adriana Wegner Araya ${ }^{\mathrm{c}}$, Pablo Bravo Figueroa ${ }^{\mathrm{d}}$, \\ Michelle Drago Thibaut ${ }^{\mathrm{e}}$, María Elisa Nalegach Romerof, Andrés Castillo Moya ${ }^{\mathrm{g}}$, Felipe Verscheure Peralta ${ }^{\mathrm{h}}$, \\ Carlos Acuña Aguirre ${ }^{\mathrm{i}}$, Franco Díaz Rubio ${ }^{\mathrm{j}}$, Paula Ortiz ${ }^{\mathrm{k}}$, Jaime Cordero ${ }^{\mathrm{l}}$, Roberto Dalmazzo Álvarez', \\ Cristian Valverde Goñi ${ }^{1}$, Mauricio Yunge Bertini ${ }^{1}$
}

\begin{abstract}
alntensivista Pediátrico. Profesor Asistente Clínico. Facultad de Medicina. Pontificia Universidad Católica de Chile. Unidad Paciente Crítico Pediátrica, Hospital Clínico Universidad Católica, Santiago. Presidente Rama Cuidados Intensivos Pediátricos, SOCHIPE

bIntensivista Pediátrica. Jefa UPC Pediátrica. Hospital Carlos Van Buren, Valparaíso. Docente Asociado Universidad de Valparaíso. Vicepresidente Rama de Cuidados Intensivos, SOCHIPE

Intensivista Pediátrica. Jefa UPC Pediátrica. Complejo Asistencial Dr. Sótero del Río. Santiago, Chile

dintensivista Pediátrico. UPC Pediátrica Hospital San Juan de Dios. Santiago, Chile

eIntensivista Pediátrica. Jefa UPC Pediátrica. Docente Universidad de Chile. Hospital Dr. Exequiel González Cortés. Santiago, Chile

IIntensivista Pediátrica. UPC Pediátrica. Hospital Dr. Exequiel González Cortés. Santiago, Chile

Intensivista Pediátrico. Profesor Asociado Adjunto. Facultad de Medicina. Jefe Unidad Académica Paciente Crítico Pediátrico. Pontificia Universidad Católica de Chile. Santiago, Chile

hIntensivista Pediátrico. UPC Pediátrica, Hospital Gustavo Fricke. Viña del Mar, Chile

Intensivista Pediátrico. Jefe UPC Pediátrica y Neonatal. Hospital Dr. Luis Calvo Mackenna. Santiago, Chile

Intensivista Pediátrico. UPC Pediátrica, Hospital El Carmen, Maipú. Santiago, Chile

kIntensivista Pediátrica. UPC Pediátrica. Hospital Dr. Roberto del Río. Santiago, Chile

IIntensivista Pediátrico. UPC Pediátrica. Clínica Las Condes. Santiago, Chile
\end{abstract}

Recibido: 25 de mayo de 2020; Aceptado para publicación: 9 de junio de 2020

\section{Resumen}

La aparición del SARS-CoV-2 a partir de diciembre de 2019 y su rápida expansión en el mundo alcanzando el estatus de Pandemia, se ha convertido en un gran desafío para los equipos de salud. Aunque la evidencia de la infección en niños aún es escasa comparada a la de los adultos, se ha hecho evidente que a nivel de la población pediátrica, la mayor parte de las veces la infección es asintomática o de curso leve, sin embargo no todos los pacientes tienen esta evolución, lo que ha motivado la discusión en los equipos de Cuidados Criticos Pediátricos respecto a cómo enfrentar estos pacientes
Palabras clave: COVID 19; SARS-CoV-2; Pediatría; Cuidados Intensivos; Tratamiento

* La presente guía fue desarrollada por el comité asesor de la Rama de Cuidados Intensivos Pediátricos de la Sociedad Chilena de Pediatría.

\section{Correspondencia:}

Christian Scheu Goncalves

cscheu@gmail.com 
con enfermedad grave. Este consenso es fruto del trabajo de la Rama de Cuidados Intensivos Pediátricos de la Sociedad Chilena de Pediatría, recogiendo la evidencia disponible al momento de la revisión más la opinión de expertos nacionales en Cuidados Intensivos Pediátricos. El propósito de estas recomendaciones, es ofrecer a los equipos que atienden a pacientes críticos pediátricos, una guía para el diagnóstico y tratamiento de pacientes que evolucionen con COVID 19 grave, que puedan ser aplicadas en todas las UPC Pediátricas de nuestro país, haciendo especial énfasis en aquellas medidas que han demostrado mayor efectividad a nivel de estudios diagnósticos, tratamiento y cuidados del personal de salud.
Keywords: COVID 19; SARS-CoV-2; Pediatrics; Critical Care; Treatment

\section{Introducción}

El SARS-CoV-2, es un nuevo Coronavirus causante de un espectro de patologías respiratorias englobadas bajo el término de COVID-19, entre las que se cuentan la neumonía grave y el síndrome de distrés respiratorio agudo. Fue descubierto, aislado y reportado por primera vez en Wuhan, China, el 7 de enero de 2020, posterior al reporte de 27 casos de síndrome respiratorio agudo de etiología desconocida en la misma localidad el 31 de diciembre de 2019.

El 30 de enero de 2020, OMS declara que el brote de COVID-19 constituye una emergencia de salud pública de importancia Internacional (ESPII) y el 11 de marzo de 2020 se declara pandemia global, dada la alta propagación del virus a nivel mundial, registrándose al 26 de abril de 2020 casi 2,9 millones de casos y 200.000 muertes en todo el mundo. En tanto en Chile, el reporte al 24 de abril de 2020 informa 12.214 casos confirmados, de los cuales el 4,4\% (540 pacientes) correspondió a menores de 15 años.

La evidencia preliminar apunta a que los casos pediátricos de infecciones por SARS-CoV-2 tendrían un curso menos grave respecto a lo descrito en adultos, con una incidencia muy baja en este grupo etario (1\%$8 \%$ de los casos diagnosticados). En pacientes hospitalizados por infecciones del tracto respiratorio inferior este virus se ha identificado en un $4 \%$ a $6 \%$ de los casos $y$ en el ambiente ambulatorio hasta en un $8 \%$ de los pacientes con patología respiratoria. Se ha reportado que en un $50 \%$ a $80 \%$ de los pacientes pediátricos se identifica un familiar infectado.

El período de incubación descrito varía entre 1 a 14 días, concentrándose principalmente entre el día 3 y 7 . Se han descrito casos desde el primer día de vida $^{5,6}$.

En los niños, los síntomas descritos son: fiebre (que puede o no estar presente), síntomas respiratorios habitualmente leves (rinitis, otitis, faringitis y laringitis), mialgias, cefalea y síntomas gastrointestinales en hasta un $40 \%$ de los pacientes (diarrea, vómitos). La infección por SARS-CoV-2 puede estar presente en pacientes asintomáticos $(9 \%)$.

La coinfección viral es frecuente (alrededor de $40 \%$ ) y acorde a la epidemiología estacional, por lo que es esperable que en niños la falla respiratoria grave sea explicable por las causas virales habituales durante epidemia estacional.

La infección severa por SARS-CoV-2 en niños es poco frecuente. Los datos reportados en China muestran que de un total de 2.143 niños con COVID-19 solamente el 5,6\% tenía una enfermedad severa definida como hipoxia y el $0,6 \%$ una falla multiorgánica o SDRA. Al momento de la edición de estas guías se ha reportado mortalidad pediátrica en Francia (16 años), Bélgica, Reino Unido, Estados Unidos, China (2 pacientes, uno un lactante de 10 meses fallecido por falla multiorgánica 4 semanas después de la admisión) 
y Colombia (preescolar de 3 años y lactante 1 mes 7 días).

\section{Alcance de la guía}

Esta guía está dirigida a profesionales del área de la salud que se desempeñan en Unidades de Paciente Critico Pediátrico, tanto públicas como privadas.

\section{Consideraciones}

Esta guía fue elaborada con la intención de establecer estándares de cuidado para el paciente pediátrico con sospecha/confirmación de COVID-19, reconociendo las actuales prácticas clínicas pediátricas aceptadas para este grupo etario en la patología respiratoria en general y asumiendo la escasa evidencia pediátrica reconocida a la fecha para esta patología en particular.

En esta guía se pone énfasis en la adopción de medidas de bioseguridad recomendadas por la OMS, CDC y MINSAL, además de recoger las recomendaciones recientemente realizadas por el grupo de Kinesiología Intensiva y Terapia Respiratoria Pediátrica nacional.

Las recomendaciones realizadas en esta guía están sujetas a cambios, acorde a la aparición de nueva evidencia en la población pediátrica.

\section{Definiciones}

Las definiciones de caso pueden variar y deben ser actualizadas de acuerdo a las directrices del MINSAL. Al momento de estas recomendaciones las definiciones ministeriales vigentes son las siguientes:

\section{Caso sospechoso}

a) Viajeros: Paciente con enfermedad respiratoria aguda que presente fiebre o al menos un signo o síntoma de enfermedad respiratoria; y con historia de viaje o residencia a país, área o territorio que reporta transmisión local de COVID-19 durante los 14 días previos al inicio de los síntomas.

O bien,

b) Contactos: Paciente con cualquier enfermedad respiratoria aguda; y con antecedentes de contacto con caso confirmado o sospechoso de COVID-19 los 14 días previos al inicio de los síntomas.

O bien,

c) Circulación Local: Paciente con infección respiratoria aguda que sea residente o se encuentre de paso en una región con circulación comunitaria de SARS-CoV-2; y que presenta fiebre $\left(37,8^{\circ} \mathrm{C}\right)$ y al menos uno de los siguientes síntomas: odinofagia, tos, mialgias o disnea.
$\mathrm{O}$ bien,

d) Vigilancia: Paciente con infección respiratoria aguda grave y que requiera hospitalización.

\section{Caso probable}

Caso sospechoso en que el análisis de laboratorio por PCR para COVID-19 resultó no concluyente.

\section{Caso confirmado}

Caso Sospechoso en que la prueba específica para COVID-19 resultó positiva.

Dentro de la patología grave provocada por SARSCoV-2, la OMS define las siguientes patologías:

\section{Neumonía grave}

Tos o dificultad respiratoria asociada y al menos uno de los siguientes:

- Polipnea ( $<2$ meses $\geq 60$ rpm; 2-11 meses $\geq 50$ rpm y 1-5 años: $\geq 40$ rpm, $>5$ años $\geq 30$ rpm) excluyendo fiebre y llanto.

- Cianosis central o saturación de $\mathrm{O}_{2}$ arterial $<90 \%$ 92\%.

- Dificultad respiratoria severa: quejidos, retracción torácica universal, aleteo nasal, cianosis, apneas intermitentes.

- Letargia o compromiso de conciencia o convulsiones, apneas.

- Incapacidad o dificultad para alimentarse, signos de deshidratación.

El diagnóstico es clínico, las imágenes de tórax pueden identificar o excluir complicaciones pulmonares.

\section{SDRA pediátrico}

- Edad: excluye pacientes con enfermedad pulmonar perinatal.

- Inicio: dentro de 7 días de producida la injuria.

- Origen del edema: falla respiratoria no explicada completamente por falla cardíaca o sobrecarga de volumen.

- Radiología: hallazgo de infiltrados nuevos compatibles con afectación aguda del parénquima pulmonar.

- Oxigenación: $(\mathrm{OI}=$ índice de oxigenación y OSI = índice de oxigenación usando $\mathrm{SpO}_{2}$ ).

\begin{tabular}{|l|c|c|c|}
\hline \multirow{2}{*}{ VMNI } & \multicolumn{3}{|c|}{ VMI } \\
\cline { 2 - 4 } & Leve & Moderado & Severo \\
\hline VMNI con doble nivel & & & \\
de soporte o CPAP & $4 \leq \mathrm{OI}<8$ & $8 \leq \mathrm{OI}<16$ & $\mathrm{OI} \geq 16$ \\
$\geq 5 \mathrm{cmH}_{2} \mathrm{O}$ a través & 0 & 0 & 0 \\
de una máscara facial & $5 \leq \mathrm{OSI}$ & $7,5 \leq \mathrm{OSI}$ & OSI $\geq 12,3$ \\
completa: $\mathrm{PaO}_{2} / \mathrm{FiO}_{2}$ & $<7,5$ & $<12,3$ & \\
$\leq 300 \mathrm{mmHH}_{\mathrm{m} \mathrm{SpO}_{2} /}$ & & & \\
$\mathrm{FiO}_{2} \leq 264$ & & & \\
\hline
\end{tabular}




\section{Sepsis pediátrica}

Sospecha de infección o infección comprobada y $\geq 2$ criterios de respuesta inflamatoria sistémica basada en la edad, de las cuales una debe ser temperatura anormal o recuento de glóbulos blancos anormal (los otros 2 criterios con taquipnea y taquicardia o bradicardia en $<1$ año).

\section{Shock séptico}

Infección grave que conduce a disfunción cardiovascular, que incluye hipotensión (presión arterial sistólica $<\mathrm{Pc}_{5} \mathrm{O}>2 \mathrm{DS}$ bajo rango normal para la edad) ó 2 o 3 de las siguientes características: estado mental alterado; taquicardia o bradicardia $(\mathrm{FC}<90 \mathrm{lpm}$ o $>160$ lpm en lactantes y FC $<70 \mathrm{lpm}$ o $>150 \mathrm{lpm}$ en niños); llene capilar prolongado ( $>2$ segundos) y pulso débil o piel caliente vasodilatada con pulsos saltones; taquipnea; piel moteada o fría o rash petequial o purpúrico; láctico aumentado, oliguria; hipertermia o hipotermia.

\section{Recomendaciones para prevención de infecciones cruzadas}

Las Unidades de Paciente Crítico Pediátrico (UPCP) deben contar con una adecuada coordinación con todos los servicios pediátricos, de manera que dispongan de flujogramas de atención que faciliten los procesos de hospitalización desde las unidades de emergencia, servicios médico-quirúrgicos pediátricos hasta la UPCP.

En las unidades de emergencia infantil se sugiere segmentar los flujos de atención de pacientes, diferenciándolos según se trate de patología respiratoria o no, en un intento por disminuir la posibilidad de contagio al interior de las instituciones de salud.

Para la hospitalización de pacientes sospechosos o confirmados COVID-19 se deben mantener los criterios de hospitalización clásica para pacientes respiratorios. La unidad de hospitalización (cuidados básicos, intermedios o intensivos) estará determinada por la condición clínica del paciente, similar a la patología respiratoria habitual.

La hospitalización de los pacientes con sospecha o confirmación de COVID-19 idealmente debiese ser en una habitación individual con puerta cerrada y baño exclusivo. Las habitaciones de aislamiento con presión negativa debieran reservarse para pacientes que se someterán a procedimientos generadores de aerosoles, con un mínimo de 6 recambios de aire por hora (ideal 12). El aire de estas habitaciones debe expulsarse directamente al exterior o filtrarse a través de un filtro de alta eficiencia. Las puertas de la sala deben permanecer cerradas y la entrada y salida a la habitación deben minimizarse. De no ser posible esto último, se podría realizar la hospitalización en una habitación individual con puerta cerrada más ventilación natural con una tasa de ventilación media por hora de al menos $160 \mathrm{l} /$ seg/paciente (mínimo 80 l/seg/paciente).

Si la demanda supera la disposición de habitaciones individuales, entonces, se pueden habilitar salas para la atención de pacientes sospechosos/confirmados COVID-19, manteniendo personal exclusivo, cumpliendo las distancias mínimas tolerables entre pacientes (mínimo 1 metro), las precauciones estándares y específicas a la situación clínica y las tasas de ventilación natural recomendadas.

La utilización de equipamiento de protección personal (EPP), debe regirse por protocolos coherentes y concordantes con lo recomendado para la prevención de transmisión de SARS-CoV-2. A continuación se detallan las consideraciones y recomendaciones más importantes:

- Las precauciones estándares serán cumplidas por todo el personal durante la atención de todos los pacientes sin distinción.

- De acuerdo a la evidencia actual la transmisión se produce por contacto directo o indirecto con gotitas generadas de la vía aérea en pacientes infectados y secreciones respiratorias.

- Se recomienda realizar simulaciones y tutoriales online respecto a la puesta y retiro de los elementos de protección personal respaldados por el Programa de Control de Infecciones de cada centro.

- Selección de EPP: el tipo de EPP va a depender de las actividades o atenciones que se desarrollarán con el paciente sospechoso o confirmado:

- Atención sin la ejecución de procedimientos generadores de aerosoles de riesgo: Precauciones estándares más precauciones de contacto y gotitas. El EPP se utilizará durante toda la atención del paciente y debe ser colocado antes de ingresar a la habitación o unidad del paciente y se retirará antes de salir de esta. El EPP considera:

- Delantal impermeable de manga larga, de apertura posterior.

- Guantes de látex o nitrilo impermeables que cubran el puño.

- Mascarilla quirúrgica, de preferencia preformada no colapsable (no mascarillas de género u de otro tipo de confección local).

- Protección ocular, antiparras o escudo facial.

- Atención de pacientes sospechosos o confirmados con ejecución de procedimientos generadores de aerosoles de riesgo: Precauciones estándares más precauciones de contacto, gotitas y aéreo. El EPP deberá ser utilizado durante toda la atención del paciente. Debe ser colocado antes de ingresar a la habitación o unidad del paciente y se retirará justo antes 
de salir de está, con la excepción del respirador o mascarilla con filtro absoluto tipo N95, FFP2 o similar que se realizará después de salir de la habitación o unidad. El EPP considera:

- Delantal impermeable de manga larga desechables, de apertura posterior. En caso de no contar con bata impermeable manga larga desechable, utilizar batas manga larga impermeables de polipropileno (quirúrgicas) que por su naturaleza son reutilizables y se encuentran disponibles por la actividad quirúrgica.

- Guantes de látex o nitrilo impermeables que cubran el puño.

- Respirador tipo N95, FFP2 o equivalente.

- Protección ocular, antiparras o escudo facial.

A la fecha, los procedimientos generadores de aerosoles con riesgo de transmisión comprobados son:

- Intubación endotraqueal.

- Broncoscopía.

- Ventilación mecánica no invasiva.

- Traqueostomía.

- Aspiración de secreciones con circuito abierto (independiente del procedimiento asociado en el cual se realice (ventilación invasiva, endoscopia digestiva alta, etc.).

- Ventilación manual antes de la intubación.

- Pronación del paciente.

- Desconexiones del ventilador mecánico invasivo.

- Reanimación cardiopulmonar.

- Procedimientos realizados con motores o turbinas de alta velocidad en la cavidad oral, micromotores, escaladores ultrasónicos y jeringas triples.

Con la información actualmente disponible en la literatura, es a la fecha incierta la contribución a la generación de aerosoles infectantes de otros procedimientos tales como, la utilización de cánula nasal de alto flujo, la administración de tratamientos nebulizados y la kinesioterapia respiratoria.

\section{Visitas y trazabilidad}

Los pacientes con sospecha/confirmados COVID-19 deben acogerse a las recomendaciones de cada centro asistencial, el cual debe considerar: la normativa nacional que establece cuarentena para los contactos COVID-19, la realidad e infraestructura local y el servicio en el cual se encontrará hospitalizado el paciente.

Mientras sea posible, se recomienda mantener la trazabilidad (registros) de los contactos intrahospitalarios (personal de salud/otros) con el paciente confirmado COVID-19, siempre en acuerdo con el comité de IAAS de cada centro.

\section{Diagnóstico por imágenes}

El diagnóstico imagenológico debe seguir inicialmente los mismos lineamientos de las infecciones respiratorias bajas, recomendándose la evaluación radiológica con radiografía de tórax AP y lateral simple (considerar que la evolución de la radiografía NO está relacionada de forma estricta con la evolución clínica).

Si hay disponibilidad de ultrasonografía pulmonar, puede ser utilizada para el seguimiento diario del compromiso pulmonar.

No se recomienda el uso de TAC pulmonar de manera rutinaria, a menos que determine alguna conducta diagnóstica-terapéutica específica. En caso de determinarse la pertinencia de esta imagen, se recomienda el respaldo de un especialista capaz de interpretarla.

La evaluación de pertinencia de TAC debe considerar el riesgo/beneficio para el paciente (irradiación) y para el ambiente hospitalario (traslado de paciente infectado hacia la unidad de imagenología).

\section{Tratamiento general}

\section{Uso de antipiréticos}

No se dispone actualmente de evidencia sólida respecto a la contraindicación del uso de ibuprofeno en COVID-19.

Se recomienda utilizar como primera opción paracetamol, pudiéndose usar posteriormente ibuprofeno (u otros AINE de uso habitual en pediatría) en caso de ser necesario.

\section{Cánula nasal de alto flujo (CNAF)}

Para pacientes sospechosos/confirmados COVID-19, se sugiere mantener las indicaciones consensuadas de uso de CNAF en pediatría, en unidades con experiencia en esta terapia ventilatoria, evitando el retraso de intubación endotraqueal en caso de presentarse la indicación para aquello.

Se recomienda establecer una monitorización estricta y evaluar constantemente los criterios clínicos de éxito o fracaso dentro de los primeros 60-90 min de iniciada la terapia, para definir la necesidad de escalar a otro tipo de soporte ventilatorio.

Si bien algunos estudios reportan que la cánula nasal de alto flujo (CNAF) no conferiría un mayor riesgo de transmisión de la enfermedad y que tendría un riesgo de contaminación ambiental similar a los sistemas de oxigenoterapia convencionales, aún la evidencia no es lo suficientemente concluyente. Por lo tanto, si se implementa frente a un escenario clínico compatible, se recomienda asegurar una adecuada fijación de la cánula a las narinas y adoptar las medidas ambientales y de protección personal para un procedimiento que 
favorece la dispersión del aire exhalado y que podría generar aerosoles.

No se debe utilizar terapias nebulizadas en CNAF y se deben evitar las desconexiones entre el circuito y la cánula para no aumentar la dispersión del aire exhalado.

No se sugiere el uso de mascarilla quirúrgica sobre la naricera de alto flujo, pues esta estrategia podría promover la retención de $\mathrm{CO}_{2}$, además del rápido humedecimiento de la mascarilla con la pérdida de su protección teórica.

\section{Ventilación mecánica no invasiva (VMNI)}

La VMNI es una terapia ventilatoria ampliamente aceptada y difundida para el manejo del paciente pediátrico que cursa con insuficiencia respiratoria aguda, dado sus reconocidos buenos resultados en este contexto.

Si bien no hay suficiente experiencia reportada acerca del uso de VMNI en niños cursando insuficiencia respiratoria aguda por COVID-19, sugerimos para estos pacientes mantener las indicaciones clásicas de esta terapia ventilatoria en unidades con experiencia en este tipo de terapia y que dispongan de una adecuada monitorización.

Una vez asegurada la correcta indicación e implementación de la VMNI, se recomienda evaluar la respuesta a esta terapia dentro de las primeras $2 \mathrm{~h}$ iniciada, con la finalidad de no retrasar la intubación si no se verifica en este plazo una respuesta favorable.

\section{Aspectos técnicos}

Por tratarse de un procedimiento establecido como generador de aerosoles, se recomienda mantener a los pacientes en VMNI sospechosos/confirmados COVID-19 en piezas individuales con presión negativa o, en su defecto, en una habitación individual con puerta cerrada más ventilación natural con una tasa de ventilación suficiente. De no disponerse de lo anterior, se puede realizar aislamientos de cohorte asegurando cumplir con los requerimientos establecidos para ello. Adicionalmente, se debe asegurar el cumplimiento estricto del uso de elementos de protección personal para procedimientos generadores de aerosoles para todo el personal que realice labores vinculadas al paciente (Ver apartados correspondientes).

Se recomienda como primera opción la utilización de interfases faciales (en su defecto oronasales) sin puerto exhalatorio en la mascarilla, para disminuir la dispersión del aire exhalado, asegurando el adecuado sello de la interfase a la cara del paciente para evitar las fugas de aire (importante además para la óptima entrega de las presiones preseteadas).

Si bien el uso de un VM convencional (ventilador pesado) con software específico para VMNI incor- porado en su sistema operativo ofrecería una mayor bioseguridad (circuito de doble rama con aire proveniente de una red central de gases en rama inspiratoria y filtro viral/bacteriano de alta eficacia al final de la rama espiratoria), tiene la limitación de no otorgar una adecuada compensación de fuga y de no sensar adecuadamente los esfuerzos inspiratorios en un paciente lactante - preescolar, por lo cual no se recomienda en niños menores de $30 \mathrm{~kg}$.

Se sugiere utilizar como primera alternativa un ventilador específico para VMNI (generador de flujo) asociado a un circuito monorama con puerto exhalatorio pasivo lateral (Ej., RT319, Fisher \& Paykel Healthcare Ltd, Auckland, New Zealand) adicionando un filtro viral bacteriano de alta eficacia (HEPA) proximal al ventilador específico para VMNI (filtra aire tomado desde el ambiente) y a la salida de puerto exhalatorio lateral. Este sistema, además, permite mantener la humidificación activa, importante para el confort del paciente y el buen desempeño del equipo.

Una alternativa frente a la no disposición del circuito monorama específico descrito anteriormente, es el uso de un VMNI específico (generador de flujo) asociado un circuito monorama clásico con un filtro HMEF (HME con filtro integrado, "Heat and Moisture Exchanger Filter") en línea intercalado entre la interfase y el puerto exhalatorio pasivo, además de un filtro viral bacteriano de alta eficacia (HEPA) proximal al ventilador específico para VMNI. El filtro HMEF reemplaza la humidificación activa. Considerando lo anterior y dado que la posición del filtro HMEF en el circuito es en serie, podría no ser bien tolerada por el paciente por la falta de una termohumedificación adecuada y por la posibilidad de aumentar la resistencia y las presiones/volúmenes administrados. Si se opta por esta alternativa se sugiere vigilar activamente la tolerancia y comodidad del paciente, vigilar las variaciones en las presiones de vía área real respecto de las programadas (volumen corriente), e idealmente, utilizarla en pacientes $>30 \mathrm{~kg}$ de peso.

Por último, si no se dispone de ninguna de las opciones antes descritas, se puede optar por un circuito monorama para ventiladores específicos de VMNI con válvula exhalatoria pasiva o activa en línea utilizando un HMEF entre el paciente y la válvula exhalatoria, asumiendo las mismas limitaciones ya descritas en la opción anterior.

Respecto a la aerosolterapia en pacientes con VMNI y sólo si esta está justificada como parte de la terapia específica se recomienda el uso de inhalaciones dosis medidas (MDI) en reemplazo de la terapia nebulizada, con el fin de evitar mayor aerosolización del aire exhalado.

Para la administración de MDI en VMNI se recomienda intercalar una aerocámara o un conector en lí- 
nea conectado en el circuito, para evitar la desconexión de la interfase y con ello la dispersión del aire exhalado. Para cada configuración de VMNI propuesta se sugiere lo siguiente:

- En la configuración de VMNI doble rama, instalar la aerocámara o conector en línea en la vía inspiratoria del respirador.

- En la configuración de VMNI monorama con portal exhalatorio lateral y filtro, instalar la aerocámara o conector en línea distal al portal exhalatorio y filtro, teniendo la precaución de cubrir el portal exhalatorio durante la administración de los puff para evitar el escape del medicamento a través de él.

- En la configuración de VMNI monorama con filtro HMEF en serie, instalar la aerocámara o conector en línea proximal al filtro, esto es, entre la interfase y el filtro.

Se recomienda que todos los procedimientos que requieran desconexión del paciente del VMNI sean los más breve posible (aspiración de secreciones, prevención de UPP) y se realicen con apagado del equipo previo a la desconexión para evitar mayor dispersión de aerosoles.

\section{Recomendaciones para la instrumentalización de la vía aérea}

El manejo de vía aérea de los pacientes sospechosos/ confirmados COVID-19 debe estar a cargo del equipo con mayor experiencia y entrenamiento en vía aérea.

En pacientes sospechosos/confirmados COVID-19, se recomienda aplicar las indicaciones habituales de intubación endotraqueal para pacientes con insuficiencia respiratoria aguda. Se debe procurar, en lo posible, la identificación precoz de esta condición con el objetivo de evitar situaciones de emergencia y permitir la organización adecuada del equipo.

Los equipos de vía aérea designados en cada centro para la realización de la intubación endotraqueal debiesen seguir las siguientes recomendaciones.

- Los equipos recibirán capacitación sobre uso y retiro de EPP, además de realizar simulaciones.

- Definición previa de roles durante el procedimiento de intubación.

- Implementación de un chequeo de equipamiento y personal.

Para el procedimiento de intubación propiamente tal se recomienda:

- Restringir el número de personas asistiendo el procedimiento.

- Realizar el chequeo del equipamiento y del personal involucrado en el procedimiento (previamente predefinido).
- Uso de EPP de acuerdo con lo descrito anteriormente respecto a procedimientos generadores de aerosoles.

- Verificación de monitorización cardiorrespiratoria.

El objetivo principal es desarrollar una intubación endotraqueal y una extubación segura, evitando tos y aerolización de secreciones. Se puede realizar secuencia de intubación rápida o una secuencia de intubación rápida modificada, considerando una ventilación manual gentil a presiones $<12 \mathrm{cmH}_{2} \mathrm{O}$, que permita oxigenación y movilidad del tórax y menos desaturaciones y eventos cardiovasculares.

Durante la preoxigenación considerar máscara facial adecuada para el paciente, si se requiere ventilación a presión positiva utilizar el mínimo de presiones necesarias, asegurando que la bolsa de insuflación tenga conectado un filtro de alta eficacia (HEPA). Además, es recomendable asegurar con dos manos la máscara a la cara del paciente con un segundo operador comprimiendo la bolsa para disminuir al máximo la fuga de aerosoles.

Se recomienda considerar intubación bajo cobertura plástica transparente como barrera física.

De ser posible y contar con el recurso y entrenamiento necesario, se sugiere intubar bajo videolaringoscopía.

Se sugiere utilizar tubo orotraqueal con cuffe insuflar a presiones $<$ a $25 \mathrm{cmH}_{2} \mathrm{O}$. Posterior a la intubación se sugiere mantener esta misma presión, evitando las desconexiones. De requerirse desconexión, se debe pinzar el tubo endotraqueal y reconectar lo antes posible.

\section{Manejo ventilatorio}

El uso de ventilación mecánica invasiva constituye una herramienta fundamental en el manejo de pacientes COVID-19 con insuficiencia respiratoria grave, definida para pediatría según los parámetros de consenso de PALICC/PEMVECC.

Debido al bajo número de pacientes pediátricos críticos y a la falta de suficientes publicaciones pediátricas relacionadas con la falla respiratoria grave y su mecánica pulmonar, las sugerencias realizadas a continuación están basadas en los consensos PALICC 2015, PEMVECC 2017, PEMVECC-COVID19 2020 y la experiencia en adultos con falla respiratoria grave.

Por otra parte, es recomendable que al menos al inicio de la conexión y luego durante la evolución del paciente, se realicen mediciones de mecánica pulmonar, que al menos deben incluir la compliance o distensibilidad pulmonar estática (Cest) del paciente $($ Cest $=\mathrm{Vc}$ / (Ppl-PEEP), con medición de la $\mathrm{P}^{\circ} \mathrm{Plateau}(\mathrm{Ppl})$ y el Driving Pressure $(\mathrm{DP}=(\mathrm{VC} / \mathrm{Cest})=\mathrm{Ppl}-\mathrm{PEEP})$. 
Es importante destacar que la gran mayoría de los pacientes pediátricos que requerirán ventilación mecánica por COVID-19, se debieran comportar como neumonía viral más que como SDRA. En ese escenario, el manejo ventilatorio será similar al de otras causas virales de neumonía grave y requerirán de parámetros ventilatorios más gentiles. Las recomendaciones que siguen van orientadas a los pacientes con falla respiratoria con comportamiento como SDRA.

Al momento de escribir esta guía, nuestras sugerencias corresponden a las siguientes:

- Modalidad ventilatoria: No recomendamos un modo sobre otro. Es importante que la modalidad elegida considere las prácticas habituales de cada una de las unidades, considerando los beneficios y riesgos de cada una.

- Volumen corriente (Vc): Vc deberá estar en función de la compliance o distensibilidad pulmonar del paciente. El volumen corriente recomendado es de:

- $\quad 5$ a $8 \mathrm{ml} / \mathrm{kg}$ de peso corporal ideal en pacientes con distensibilidad conservada.

- 3-6 ml/kg para aquellos pacientes con distensibilidad muy disminuida.

- Se debe evitar más de $10 \mathrm{ml} / \mathrm{kg}$.

- PEEP: Se recomienda ajustar la PEEP de acuerdo a las recomendaciones PALICC/PEMVECC.

- El PEEP inicial debe ser de $\pm 10 \mathrm{~cm} \mathrm{H}_{2} \mathrm{O}(8 \mathrm{a}$ $12 \mathrm{cmH}_{2} \mathrm{O}$ ), debiendo titularse de acuerdo a la oxigenación, la respuesta hemodinámica y a la distensibilidad del sistema respiratorio (presión Plateau y driving presure).

- En pediatría no existe un método estándar para definir el PEEP por lo que se recomiendan las tablas $\mathrm{PEEP} / \mathrm{FiO}_{2}$ del ARDS Network.

- En los pacientes que requieran de PEEP más alto, se sugiere mantener una monitorización rigurosa de complicaciones con $\mathrm{Rx}$ de tórax y control hemodinámico estricto ante la mayor posibilidad de deterioro.

- Presión Plateau (Ppl) y Driving Pressure (DP): Se recomienda seguir las sugerencias PALICC/PEMVECC:

- Mantener Presión plateau < 30, recomendándose $<28 \mathrm{~cm} \mathrm{H}_{2} \mathrm{O}$.

- En situaciones de muy baja distensibilidad se podría tolerar hasta 29 a $32 \mathrm{~cm} \mathrm{H}_{2} \mathrm{O}$.

- Mantener Driving pressure $\leq 15$.

- Oxigenación: En ausencia de nueva evidencia, se sugiere mantener las recomendaciones PALICC/ PEMVECC respecto a titular las saturaciones $\mathrm{y}$ $\mathrm{FiO}_{2}$ de acuerdo a la gravedad del paciente. El con- senso es utilizar la $\mathrm{FiO}_{2}$ más baja necesaria para lograr una saturación que asegure una adecuada $\mathrm{DO}_{2}$, no siendo necesarias saturaciones $>96-97 \%$ :

- $\quad \geq 92 \%$ para SDRA leve con PEEP $<10 \mathrm{cmH}_{2} \mathrm{O}$.

- 88\%-92\% para SDRA con PEEP $\geq 10 \mathrm{cmH}_{2} \mathrm{O}$.

- Monitorizar acidosis metabólica, láctico en ascenso y $\mathrm{SvO}_{2}$ de cava superior.

- Hipercapnia permisiva: Se recomienda aceptar valores de hipercapnia permisiva (valores mayores de $\mathrm{pCO}_{2}$ ) de acuerdo a la gravedad del paciente, con $\mathrm{pH}$ sanguíneo entre 7,15 y 7,30, siempre que no existan contraindicaciones tales como hipertensión endocraneana, hipertensión pulmonar o inestabilidad hemodinámica.

- Posición prono: En base a la evidencia actual en pacientes con SDRA adultos y la opinión de expertos en pacientes COVID-19 positivos, la posición prono se recomienda en pacientes con $\mathrm{PaO}_{2} / \mathrm{FiO}_{2}$ $<150\left(\mathrm{PaO}_{2} / \mathrm{FiO}_{2}<150\right.$; IO $\geq 12$; ISO $\left.\geq 10\right)$. No existe evidencia de su utilidad y uso de regla en pacientes pediátricos. De utilizar, se recomienda uso temprano y prolongado (12 a $18 \mathrm{~h}$ ).

En pacientes sospechosos/confirmados COVID-19 en VMI se recomienda seguir los lineamientos establecidos para la población pediátrica en PALICC y PEMVECC respecto a:

- Aportar líquidos totales para mantener un adecuado volumen intravascular, perfusión de los órganos blanco y la entrega óptima de oxígeno.

- Monitorizar y titular la administración de fluidos para un adecuado volumen intravascular evitando los balances hídricos positivos, una vez realizada la reanimación y estabilización.

- Considerar el uso de bloqueadores neuromusculares si la sedación es insuficiente para lograr una ventilación mecánica efectiva, evitando las asincronías.

- Se recomienda utilizar la menor dosis efectiva.

- Si no se dispone de monitorización del nivel de bloqueo neuromuscular, realizar una suspensión diaria de la infusión para permitir el monitoreo del nivel de sedación y disminuir las complicaciones secundarias al uso prolongado de bloqueo neuromuscular.

\section{Aspectos técnicos}

Considerando que los gases provienen filtrados desde una red central al ventilador mecánico, se recomienda el uso de un filtro viral/bacteriano de alta eficacia al final de la rama espiratoria del circuito ventilatorio, dejando como decisión de cada centro la adición de un filtro viral/bacteriano de alta eficacia al inicio de la rama inspiratoria del circuito ventilatorio. 
Se recomienda realizar cambios de filtro viral/bacteriano de alta eficacia cada 24 h o ante disfunción contaminación visible del mismo.

Se sugiere utilizar humidificadores activos servocontrolados junto a circuitos con alambre calefactor interno de doble rama, de preferencia desechables, para disminuir la condensación del circuito. De no disponerse de lo anterior, se podría optar por una humidificación pasiva por medio de filtros intercambiadores de calor y humedad, asumiendo una posible disminución en la efectividad de la terapia ventilatoria (Ej., aumento del espacio muerto instrumental).

Se recomienda el uso de sistemas de aspiración cerrada de secreciones para evitar la generación de aerosoles al abrir el circuito ventilatorio para realizar este procedimiento. De no ser posible la disposición de circuitos cerrados y frente a la necesidad de realizar aspiración de secreciones, se recomienda: adoptar las medidas de protección personal establecidas para un procedimiento generador de aerosoles, pinzar el tubo endotraqueal, poner el ventilador mecánico en modo inactivo antes de la desconexión, asistir la respiración con sistema de ventilación manual con filtro HEPA incorporado, realizar la aspiración (por el menor tiempo posible), reconectar el circuito del ventilador y salir del modo inactivo retirando finalmente la pinza del tubo endotraqueal. Es fundamental que la frecuencia de estos procedimientos se disminuya al mínimo posible, siempre que esto no ponga en riesgo al paciente.

Respecto a las terapias con broncodilatadores inhalados, no se dispone de evidencia de su beneficio en cuadros graves de COVID-19 o pARDS. Considerarlas sólo frente a: presencia de signología obstructiva moderada-severa, paciente con antecedentes de asma o hiperreactividad bronquial y siempre considerando riesgo/beneficio de la terapia.

Si alguna de las condiciones anteriores está presente, se recomienda la administración de inhaloterapia mediante una cámara espaciadora y dispositivos MDI (inhalador de dosis medida) para mejorar el depósito de las partículas.

En caso de no disponerse de cámaras espaciadoras, se sugiere aumentar el número administración de dosis medida (6-8 puff).

No se recomienda el uso de nebulizaciones en VMI dada la generación de aerosoles de estas, si bien la tecnología de administración de medicamentos en aerosol de malla vibrante en circuito cerrado podría ser una alternativa plausible.

De ser necesaria la obtención de muestras para diagnóstico en pacientes intubados, se recomienda tomarlas por aspirado endotraqueal versus secreciones nasofaríngeas, adoptando siempre las medidas de protección personal recomendadas para un procedi- miento generador de aerosoles. No se recomienda realizar fibrobroncoscopía para la obtención de un lavado broncoalveolar, a menos que sea estrictamente necesario y tengan implicancias en el manejo del paciente.

\section{VAFO}

Considerando la evidencia actual no podemos realizar una recomendación específica respecto a su uso. Podría utilizarse como terapia de rescate en aquellos centros que tengan familiaridad con esta modalidad ventilatoria, utilizando los mismos criterios en relación a falla respiratoria refractaria.

Considerar el alto riesgo de aerosolización de esta terapia, Se recomienda extremar las medidas de protección ambiental y personal.

\section{ECMO}

A la fecha no existen diferencias de las actuales indicaciones y contraindicaciones generales de ECMO para pacientes COVID-19 con falla respiratoria catastrófica refractaria.

Dada la ausencia de literatura de ECMO en pacientes pediátricos COVID-19 al momento de la redacción de este documento, se sugiere la discusión, caso a caso, considerando la situación del paciente, patologías de base y la disponibilidad de ECMO en cada centro o región geográfica y según las condiciones epidemiológicas del brote de COVID-19 al momento del análisis.

\section{Terapias farmacológicas específicas}

\section{Antibióticos}

Considerar precozmente inicio de antibióticos en caso de sospecha de infección bacteriana, y ajustar terapia con resultado de cultivos.

\section{Corticoides sistémicos}

Los corticoides sistémicos no están recomendados de forma general. Estudios previos en pacientes con SARS, MERS e incluso gripe han demostrado que no tienen efectos beneficiosos en incluso ha retrasado el aclaramiento del virus. Sin embargo, su uso podría justificarse en casos de:

- Obstrucción bronquial.

- Deterioro rápido de imagen torácica y SDRA.

- Shock séptico.

- Encefalitis/encefalopatía, síndrome hematofagocítico, entre otras complicaciones graves.

\section{Gamaglobulina}

Se ha reportado el uso de inmunoglobulina endovenosa en varias series de pacientes adultos con COVID-19 de evolución grave por su acción inmunomoduladora, sin embargo, no hay evidencia de su real utilidad. 


\section{Tocilizumab}

El tocilizumab es un anticuerpo monoclonal IgG1 recombinante humanizado anti receptor de interleucina-6. Se ha demostrado que tocilizumab inhibe la señalización mediada por IL-6Rs e IL-6Rm. De acuerdo a información disponible actualmente se ha objetivado que existe una alta concentración de citoquinas en pacientes críticos infectados con COVID-19. El uso de tocilizumab se ha planteado como forma de evitar la tormenta de citoquinas en el SDRA de estos pacientes. Actualmente, no existe evidencia sólida de ensayos clínicos publicados que evalúen la seguridad o la eficacia de tocilizumab para el tratamiento de COVID-19, incluido el tratamiento de las tormentas de citoquinas asociadas. Es necesario considerar que la información de su uso en niños para esta indicación es casi nula.

Considerar como tratamiento en pacientes con desregulación de la respuesta inflamatoria o con sospecha de síndrome hemofagocítico (dímero-D aumentado, ferritina aumentada, fibrinógeno disminuido, hipertrigliceridemia, leucopenia, trombocitopenia.

\section{Hidroxicloroquina}

Medicamento utilizado en forma habitual como antimalárico, posee actividad contra varios virus incluidos el coronavirus. La actividad in vitro contra SARS-CoV-2 es más potente que la cloroquina y poseería mejor perfil de seguridad. La hidroxicloroquina también posee efectos inmonomoduladores como la reducción de la producción de citoquinas, especialmente IL-1 e IL-6.

Su uso en etapas tempranas tendría efecto in vitro contra SARS-CoV-2, sin embargo, no existe evidencia suficiente adulto/pediátrica respecto a la real eficacia de hidroxicloroquina para recomendar su uso de rutina.

Podría considerarse su uso en pacientes graves, dado que es una terapia más costo-efectiva y con relativos menos efectos adversos respecto de las otras alternativas propuestas.

En caso de utilizarse, se sugiere controlar ECG previo al inicio de la terapia y $24-48 \mathrm{~h}$ post inicio.

\section{Azitromicina}

Un estudio mostró que el tratamiento de hidroxicloroquina asociado a azitromicina podría disminuir la carga viral sinérgicamente. Sin embargo, el estudio tenía limitaciones importantes y un número pequeño de pacientes. Adicionalmente, existe evidencia creciente en adultos de los efectos adversos a nivel cardiovascular (arritmias) del uso de azitromicina usado concomitante con hidroxicloroquina. Basados en lo anterior, no parece recomendable el uso de azitromicina en este contexto.

\section{Terapia antiviral específica (lopinavir/ritonavir} [Kaletra ${ }^{\circledR}$, Remdesivir)

El uso de estos antivirales tendría algún beneficio utilizados precozmente en la evolución de la enfermedad, ya que disminuirían la carga viral. Sin embargo, a la fecha no existe evidencia adulto/pediátrica suficiente respecto a la eficiencia de algún antiviral específico para hacer una recomendación de manejo.

El lopinavir es un inhibidor de la proteasa antirretroviral utilizado en combinación con Ritonavir para el tratamiento de la infección por VIH. Tendría actividad in vitro contra SARS-CoV y MERS-CoV. Actualmente se encuentra en evaluación en un protocolo de tratamiento planificado por la OMS y en el ensayo REMAPCAP.

Respecto a Remdesivir, corresponde a un análogo de nucleótido no autorizado que interfiere con la polimerización del ARN del virus. Fue desarrollado inicialmente como tratamiento para la enfermedad del virus del Ébola, pero presenta también actividad in vitro frente a éste y otros virus, incluyendo el coronavirus. Es uno de los fármacos que se investigarían a mayor escala por la OMS. Actualmente, no se dispone de este medicamento en Chile.

\section{Interferon- $\alpha 2 b$ nebulizado}

Existe escasa evidencia con esta terapia farmacológica en COVID-19, ha sido utilizada en bronquiolitis, neumonías virales, enfermedad pie-mano-boca, SARS y otras infecciones virales. Podría reducir la carga viral y usada precozmente y podría ayudar a reducir los síntomas y a acortar el curso de la enfermedad. Sin embargo, su administración aumentaría riesgo de aerosolización.

No es una terapia que actualmente se ajuste a la realidad nacional.

\section{Plasma de pacientes convalecientes}

Existen reportes de casos en adultos respecto al uso de plasma de pacientes convalecientes en casos graves de COVID-19, con resultados favorables en cuanto a mejoría en sintomatología, parámetros ventilatorios, parámetros de laboratorio y hallazgos radiológicos. Aún es insuficiente la evidencia en adultos e inexistente en pediatría para recomendar su uso. Podría considerarse en casos graves según disponibilidad.

\section{Terapia anticoagulante}

A raíz de la creciente evidencia de un estado protrombótico presente en los pacientes adultos con COVID-19 grave se ha extendido cada vez más la recomendación de expertos acerca de usar terapia anticoagulante en estos pacientes. 


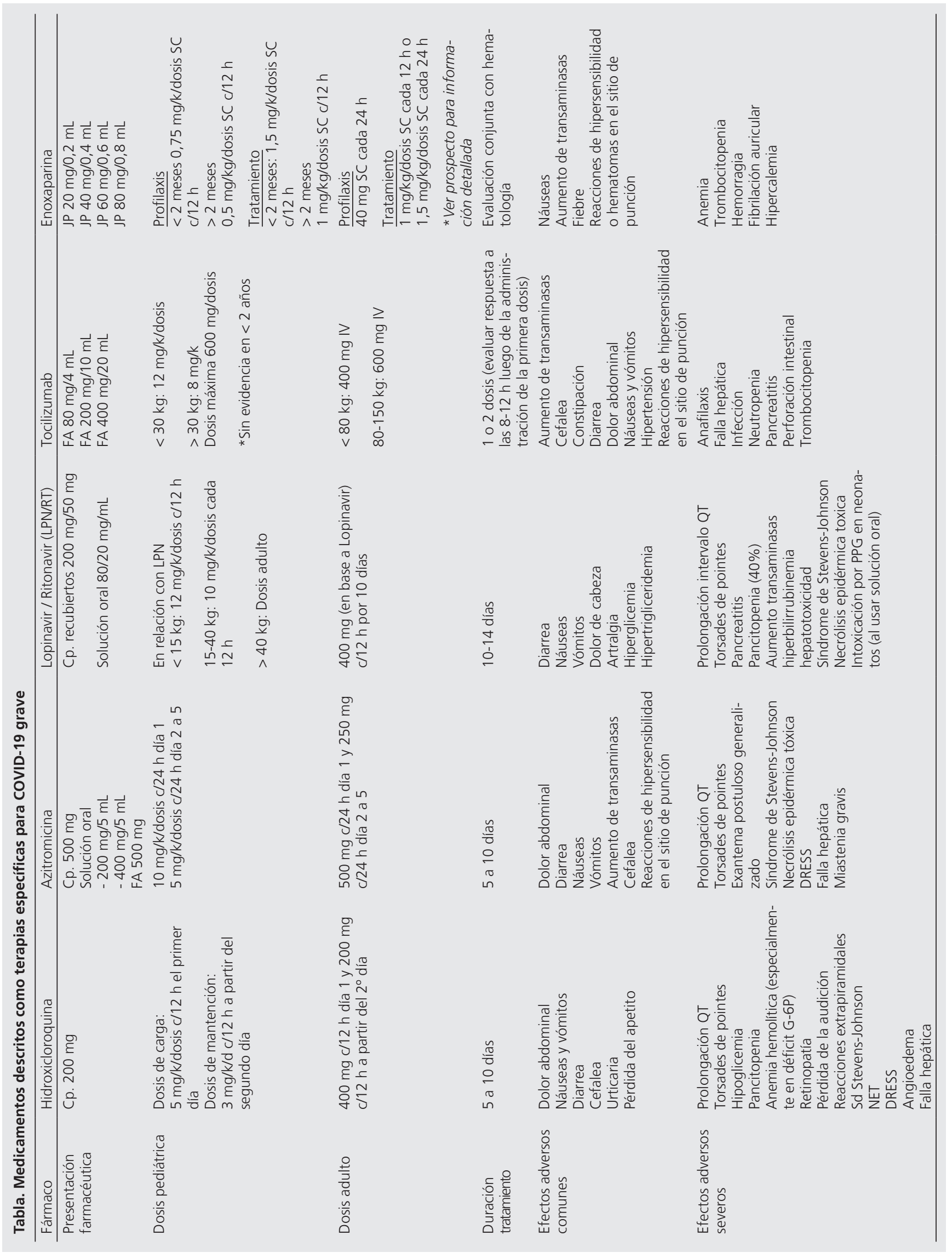




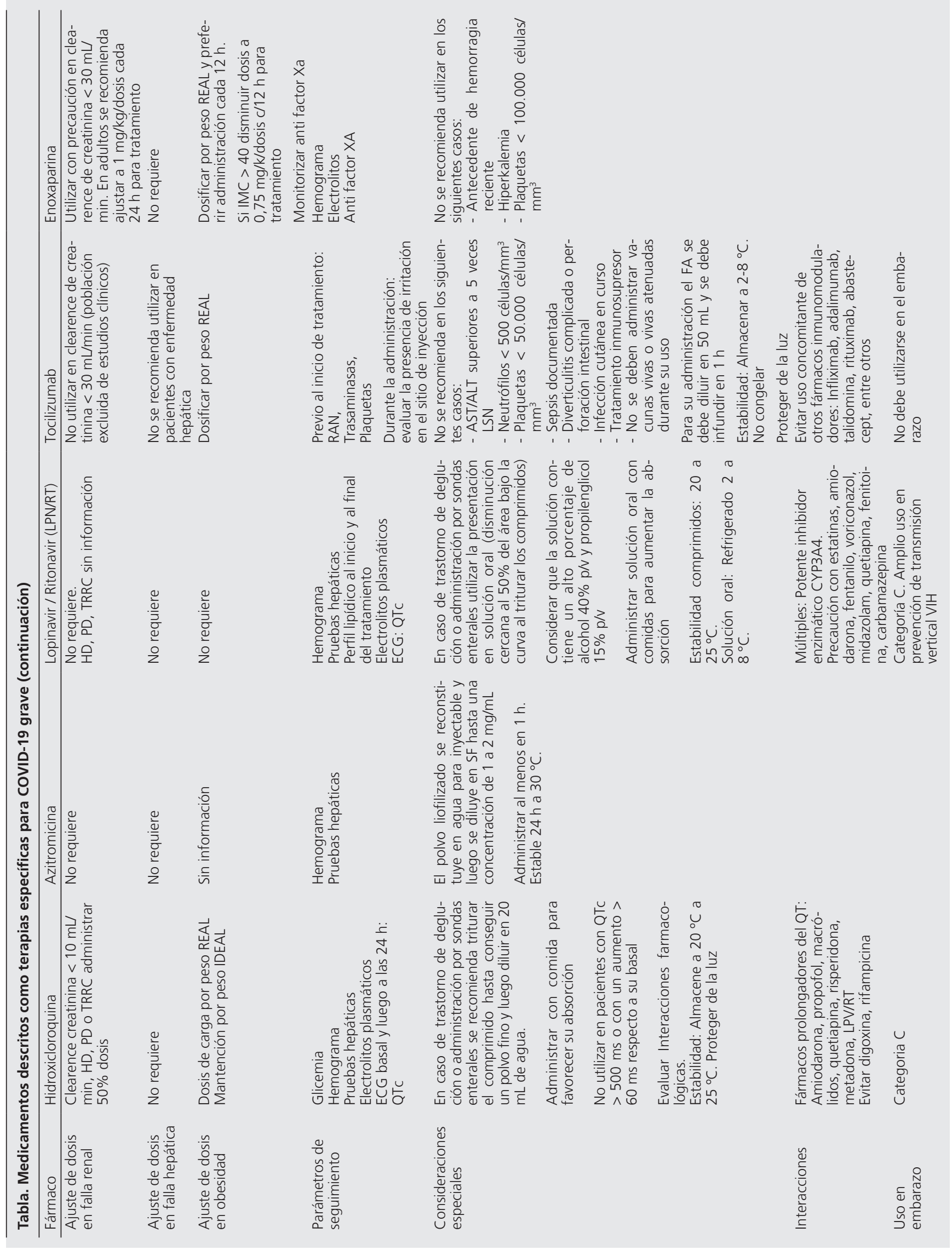


Aún hay escasa evidencia en adultos, pero parecería existir una mejor evolución en los pacientes tratados con heparina, especialmente de bajo peso molecular, en el subgrupo de pacientes graves con elevaciones muy significativas de productos de degradación del fibrinógeno, dímero D y/o criterios de coagulación intravascular diseminada.

No existe evidencia pediátrica respecto al uso de anticoagulación en COVID-19, pero ante el escenario de un estado protrombótico en un paciente grave, puede considerarse el uso de heparina de bajo peso molecular.

\section{Recomendación farmacológica}

En síntesis, y de acuerdo a la literatura y recomendaciones actualmente vigentes recomendamos para el paciente COVID-19 grave (falla respiratoria grave, SDRA, shock, encefalopatía, SDOM):

- Iniciar antibióticos en caso de sospecha de infección bacteriana acorde a la epidemiología local

- Iniciar Oseltamivir mientras se descarta infección por virus influenza

- Iniciar hidroxicloroquina oral $5 \mathrm{mg} / \mathrm{kg} /$ dosis cada $12 \mathrm{~h}$ por 2 dosis el primer día y luego $3 \mathrm{mg} / \mathrm{kg} / \mathrm{dosis}$ cada $12 \mathrm{~h}$ por 4 días más (se puede extender hasta 10 días). Dosis máxima $400 \mathrm{mg}$ cada $12 \mathrm{~h}$ el primer día por 2 dosis y $200 \mathrm{mg}$ cada $12 \mathrm{~h}$ el resto.

- Considerar el uso de tocilizumab en casos graves con evidencias de elevación de citoquinas (IL-6), ferritina o criterios de activación macrofágica.

- Considerar el uso de corticoides en casos de shock refractario a catecolaminas, SDRA en etapas tardías en ausencia de infección por influenza, u otras indicaciones de corticoides distintas de COVID-19.
- Considerar el uso de heparina de bajo peso molecular en pacientes pediátricos con COVID-19 de evolución grave y elevación del dímero $\mathrm{D}$, asociado a alto riesgo de trombosis. Se sugiere monitorización estricta de la coagulación y el apoyo de hematología para el seguimiento de estos pacientes.

- Considerar el uso de plasma de pacientes convalecientes en casos graves y según disponibilidad.

\section{Optimización de cupos críticos a adultos}

Se recomienda ampliar el rango etario para la atención de pacientes críticos pediátricos acorde a los requerimientos locales de cada centro.

Se recomienda el manejo integral del paciente adulto por parte del equipo pediátrico, manteniendo siempre una supervisión/interconsultoría de los especialistas en patología de adultos.

Se recomienda que el personal de salud de UPCP esté disponible para apoyar unidades de adultos en caso de ser necesario (sin desproteger la unidad pediátrica).

\section{Manejo de personal UPCP}

Implementar políticas de rotación del personal de salud para disminuir exposición e intentar con ello mantener un equipo de profesionales activos, con disminución de exposición y resguardo en domicilio.

\section{Conflicto de interés}

Los autores declaran no tener conflicto de intereses.

\section{Referencias}

1. National Health Commission of People's Republic of China. En: https:// www.nhc.gov.cn/xcs/yqfkdt/202001/ a53e6df293cc4fob5a16ddf7b6b2b31.

2. Coronavirus disease 2019 (COVID-19) Situation Report-97. En: https:// www.who.int/docs/defaultsource/coronaviruse/situationreports/20200426-sitrep-97-covid-19. pdf?sfvrsn=d1c3e800_6.

3. Informe epidemiológico enfermedad por COVID-19. Chile 24-04-2020. Departamento de epidemiología. Ministerio de Salud, chile. En: https://www.minsal.cl/wp-content/ uploads/2020/04/Informe_EPI_24042020. pdf

4. Zimmermann, Petra MD, PhD, Curtis,
Nigel FRCPCH, PhD. Coronavirus Infections in Children Including COVID-19. Pediatr Infect Dis J. 2020;39:355-68.

5. Wie Xia, Jianbo Shao, Yu Guo, et al. Clinical and CT features in pediatric patients with COVID-19 infection: Different points from adults. Pediatric Pulmonology. 2020;1-6.

6. Sinha I, Harwood R, Semple MG, et al. COVID-19 infection in children. Lancet Respir Med. 2020 May;8(5):446-447. doi: 10.1016/S2213-2600(20)30152-1. Epub 2020 Mar 27.

7. Lu X, Zhang L, Du H, et al. SARS-CoV-2 Infection in Children. N Engl J Med. 2020;382(17):1663-5. doi: 10.1056/ NEJMc2005073. Epub 2020 Mar 18.

8. Ordinario B51 Nº933, Subsecretaría de Salud Pública, Subsecretaría de Redes
Asistenciales. Actualización de definición de caso sospechoso para vigilancia epidemiológica ante brote de COVID-19 en China. Ministerio de Salud de Chile, División de Planificación Sanitaria, Departamento de Epidemiología, 23 de marzo de 2020.

9. Clinical management of severe acute respiratory infection (SARI) when COVID-19 disease is suspected. Interim guidance. WHO. 13 March 2020. En: https://www.who.int/emergencies/ diseases/novel-coronavirus-2019/ technical-guidance

10. China National Health Comission. Chinese Clinical Guidance for COVID-19 Pneumonia Diagnosis and Treatment (7th edition). En: http://kjfy.meetingchina.org/ msite/news/show/cn/3337.html\#

11. Protocolo de uso de equipos de 
protección personal en la prevención de transmisión COVID-19-SARS-CoV-2 (precauciones adicionales de transmisión por gotitas y contacto). Subsecretaría de Redes Asistenciales División de Gestión de la Red Asistencial Departamento de Calidad y Seguridad de la atención. Circular 01, 18 de marzo de 2020.

12. Interim Infection Prevention and Control Recommendations for Patients with Suspected or Confirmed Coronavirus Disease 2019 (COVID-19) in Healthcare Settings. Update April 13, 2020. En: https://www.cdc.gov/coronavirus/2019ncov/hcp/infection-controlrecommendations.html

13. Using Personal Protective Equipment (PPE). En: https://www.cdc.gov/ coronavirus/2019-ncov/hcp/using-ppe. html / Prevención y control de infecciones durante la atención sanitaria de casos en los que se sospecha una infección por el nuevo coronavirus ( $\mathrm{nCoV}$ ) Orientaciones provisionales. 25 de enero de 2020. En: https://apps.who.int/iris/bitstream/hand le/10665/330685/9789240001114-spa.pdf

14. Sobre algunas medidas para la protección del personal de salud en el contexto de la atención en establecimientos de salud durante la pandemia de COVID-19. Subsecretaría de Redes Asistenciales División de Gestión de la Red Asistencial Departamento de Calidad y Seguridad de la atención. Circular 04, 21 de abril de 2020.

15. Interim Clinical Guidance for Management of Patients with Confirmed Coronavirus Disease (COVID-19). Centers for Disease Control and Prevention. March 2020. En: https://www. cdc.gov/coronavirus/2019-ncov/hcp/ clinical-guidance-management-patients. html

16. Khemani RG, Smith LS, Zimmerman JJ, Erickson S, Pediatric Acute Lung Injury Consensus Conference G. Pediatric acute respiratory distress syndrome: definition, incidence, and epidemiology: proceedings from the Pediatric Acute Lung Injury Consensus Conference. Pediatr Crit Care Med. 2015;16(5 Suppl 1):S23-40.

17. Guía de Recomendaciones para la implementación de soporte respiratorio pediátrico en COVID-19. Kinesiología Intensiva y Terapia Respiratoria Pediátrica de Chile. Marzo 2020.

18. Alhazzani W, Moller M, Arabi Y, et al. Surviving Sepsis Campaign: Guidelines on the Management of Critically Ill Adults with Coronavirus Disease 2019 (COVID-19). Intensive Care Med. 2020 Mar 28. doi: 10.1007/s00134-020-060225.

19. Kneyber MCJ, Luca D, Calderini E, et al. Recommendations for mechanical ventilation of critically ill children from the Paediatric Mechanical Ventilation Consensus Conference (PEMVECC). Intensive Care Med. 2017. 11:1-17. doi: 10.1007/s00134-017-4920-z.

20. Shen K, Yang Y, Wang T, et al. Diagnosis, treatment, and prevention of 2019 novel coronavirus infection in children: experts' consensus statement. World J Pediatr. 07 febr 2020. https://doi.org/10.1007/s12519020-00343-7.

21. Calvo C, García M, de Carlos JC, et al. Recomendaciones sobre el manejo clínico de la infección por el nuevo coronavirus SARS-CoV2. Grupo de trabajo de la Asociación Española de Pediatría. An Pediatr (Barc). 2020. https://doi org/10.1016/j.anpedi.2020.02.001.

22. Manejo clínico de pacientes con enfermedad por el nuevo coronavirus (COVID-19). SECIP. 18 de febrero de 2020. En: https://www.comv.es/wpcontent/uploads/2020/02/DT_manejo_ cl\%C3\%ADnico_COVID-19_20.02.20. pdf.

23. Interim clinical guidance for patients suspectes of/confirmed with COVID-19 in Belgium. Version 4. 19 mars 2020; En: https://epidemio.wiv-isp.be/ID/ Documents/Covid19/COVID-19_ InterimGuidelines_Treatment_ENG.pdf.

24. The Australian and New Zealand Intensive Care Society (ANZICS) COVID-19 Guidelines. Version 1. ANZICS. 16 March. En: https:// www.anzics.com.au/wp-content/ uploads/2020/03/ANZICS-COVID-19Guidelines-Version-1.pdf.

25. Maharaj R. King's Critical Care - Evidence summary Clinical Management of COVI19. 9 march 2020. En: https:// www.amedeolucente.it/public/KCC\%20 COVID19\%20Evidence\%20Summary(2). pdf.

26. Yao T-T, Qian J-D, Zhu W-Y, Wang Y, Wang G-Q. A systematic review of lopinavir therapy for SARS coronavirus and MERS coronavirus-A possible reference for coronavirus disease-19 treatment option. J Med Virol. 2020. 1-8.

27. Cao B, Wang Y, Wen D, et al. A trial of lopinavir-ritonavir in adults hospitalized with severe Covid-19. N Engl J Med. 2020;382(19):1787-99. doi: 10.1056/ NEJMoa2001282. Epub 2020 Mar 18.

28. Young BE, Xiang Ong SW, Kalimuddin $S$, et al. Epidemiologic features and clinical course of patients infected with SARS-CoV-2 in Singapore. JAMA. 2020;323(15):1488-94. doi: 10.1001/ jama.2020.3204.

29. Chen N, Zhou M, Dong X, et al. Epidemiological and clinical characteristics of 99 cases of 2019 novel coronavirus pneumonia in Wuhan, China: a descriptive study. Lancet.
2020;395(10223):507-13. doi: 10.1016/ S0140-6736(20)30211-7. Epub 2020 Jan 30.

30. Chan KS, Lai ST, Chu CM, et al. Treatment of severe acute respiratory syndrome with lopinavir/ritonavir: a multicentre retrospective matched cohort study. Hong Kong Med J. 2003;9:399-406.

31. Chu CM, Cheng VC, Hung IF, et al. Group HUSS. Role of lopinavir/ ritonavir in the treatment of SARS: initial virological and clinical findings. Thorax. 2004;59:252-6.

32. Kim UJ, Won EJ, Kee SJ, Jung SI, Jang HC. Combination therapy with lopinavir/ ritonavir, ribavirin and interferon-alpha for Middle East respiratory syndrome. Antivir Ther. 2016;21:455-9.

33. Park SY, Lee JS, Son JS, et al. Postexposure prophylaxis for Middle East respiratory syndrome in healthcare workers. J Hosp Infect. 2019;101:42-6.

34. Chen J, Ling Y, Xi XH, et al. Efficacies of lopinavir/ritonavir and abidol in the treatment of novel coronavirus pneumonia. Chin J Infect Dis. 2020;38(0):E008

35. Fang L, Karakiulakis G, Roth M. Are patients with hypertension and diabetes mellitus at increased risk for COVID-19 infection? Lancet Respir Med. 2020 Mar 11. pii: S2213-2600(20)30116-8. doi: 10.1016/S2213-2600(20)30116-8.

36. Oliver Veran, 14 de marzo de 2020. En: https://twitter.com/olivierveran/ status $/ 1238776545398923264 ? s=20$

37. AEMPS, Agencia española de medicamentos y productos sanitarios. 15 de marzo, 2020. En: https://www. aemps.gob.es/informa/notasinformativas/ medicamentosusohumano-3/2020medicamentosusohumano-3/la-aempsinforma-que-ningun-dato-indica-que-elibuprofeno-agrave-las-infecciones-porcovid-19/

38. Day M. Covid-19: ibuprofen should not be used for managing symptoms, say doctors and scientists. BMJ. 2020;368:1086. doi: 10.1136/bmj.m1086.

39. European Medicines agency. EMA advises on use non-steroidal antiinflammatories for COVID-19. Marzo 2020. EMA/136850/2020.

40. WHO A: Based on currently available information, WHO does not recommend against the use of of ibuprofen. En: https:/twitter.com/WHO/status/1240409 $217997189128 ? \mathrm{~s}=20$.

41. Alhazzani W, Möller MH, Arabi YM, et al. Surviving Sepsis Campaign: Guidelines on the Management of Critically Ill Adults with Coronavirus Disease 2019 (COVID-19). Presentado para publicación en ccmjournal.org.

42. Peng Q, Wang X, Zhang L. Findings of lung ultrasonography of novel corona 
virus pneumonia during the 2019-2020 epidemic. Intensive Care Med (2020). https://doi.org/10.1007/s00134-02005996-6.

43. Soldati G, Smargiassi A. Is there a role for lung ultrasound during the COVID-19 pandemic? J Ultrasound Med. 2020 Mar 20. doi: 10.1002/jum.15284. [Epub ahead of print].

44. Recomendaciones de Manejo Farmacológico en Pacientes Pediátricos con Infección por SARS-CoV-2 (COVID-19). Sociedad Chilena de Infectología (SOCHINF) y Rama de Cuidados Intensivos Pediátricos de Chile. Abril 2020

45. Guía de Recomendaciones para la Implementación de Soporte Respiratorio Pediátrico en COVID-19. Kinesiología Intensiva y Terapia Respiratoria Pediátrica de Chile. Marzo 2020.

46. Borba MGS, Val FFA, Sampaio VS, et al. Effect of High vs Low Doses of Chloroquine Diphosphate as Adjunctive Therapy for Patients Hospitalized With Severe Acute Respiratory Syndrome Coronavirus 2 (SARS-CoV-2) Infection:
A Randomized Clinical Trial. JAMA Netw Open. 2020;3(4):e208857. doi: 10.1001/ jamanetworkopen.2020.8857.

47. Luo P, Liu Y, Qiu L, et al. Tocilizumab treatment in COVID-19: A single center experience. J Med Virol. 2020;92(7):8148. doi: 10.1002/jmv.25801. Epub 2020 Apr 15.

48. Zhang X, Song K, Tong F, et al. First case of COVID-19 in a patient with multiple myeloma successfully treated with tocilizumab. Blood Adv. 2020;4(7):1307-10. doi: 10.1182/ bloodadvances.2020001907.

49. Michot JM, Albiges L, Chaput N, et al. Tocilizumab, an anti-IL6 receptor antibody, to treat Covid-19-related respiratory failure: a case report. Ann Oncol. 2020;31(7):961-4. doi: 10.1016/j. annonc.2020.03.300. Epub 2020 Apr 2.

50. Duan K, Liu B, Li C, et al. Effectiveness of convalescent plasma therapy in severe COVID-19 patients. Proc Natl Acad Sci USA. 2020;117(17):9490-9496. doi: 10.1073/pnas.2004168117. Epub 2020 Apr 6.

51. Zhang B, Liu S, Tan T, et al. Treatment
With Convalescent Plasma for Critically Ill Patients With SARS-CoV-2 Infection. Chest. 2020;S0012-3692(20)30571-7. doi: 10.1016/j.chest.2020.03.039.

52. Shen C, Wang Z, Zhao F, et al. Treatment of 5 Critically Ill Patients With COVID-19 With Convalescent Plasma. JAMA. 2020;323(16):1582-9. doi: 10.1001/ jama.2020.4783.

53. Tang N, Bai H, Chen $\mathrm{X}$, et al. Anticoagulant treatment is associated with decreased mortality in severe coronavirus disease 2019 patients with coagulopathy. J Thromb Haemost. 2020;18(5):1094-9. doi: 10.1111/jth.14817. Epub 2020 Apr 27.

54. Shen K-L, Yang Y-H, Jiang R-M, et al. Updated diagnosis, treatment and prevention of COVID-19 in children: experts' consensus statement (condensed version of the second edition). World J Pediatr. 2020;1-8. doi: 10.1007/s12519020-00362-4.

55. Muñoz D. Recomendaciones para el uso de terapias farmacológicas contra COVID-19. División de farmacia clínica, Soc Chil de Med Intensiva, 20 de marzo de 2020. 